\title{
10 Tips for Better Instagram Posts
}

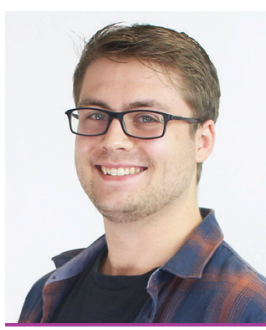

Will Cowan

As a Content Creator for Marketing4ECPs, Will Cowan writes and produces videos for eye care practices all across North America. Contact Will at marketing4ecps.com.

I nstagram is one of the most popular social media platforms, with nearly 500 million users signing in every day. But that sea of voices presents the challenge every optometric practice must deal with: standing out and making an impact. Not every image has a story worth telling, so let's take a look at 10 essential tips to get your Instagram posts to stand above the rest.

\section{Have a plan}

The first thing you need to do when you start posting on Instagram is to have a solid content plan. This plan will include the photos, images, and videos you want to feature on your feed, the aesthetic that will define your brand, and the frequency with which you post. Setting this groundwork will help keep your story coherent, relevant and on-brand.

\section{Post at the right time}

Believe it or not, there are better times to post! Generally speaking, it's best to post between 11:00 am - 1:00 pm and 7:00 - 9:00 pm, when people are on their breaks or off work.

\section{Prioritize video content}

Photos are great, but videos are better. Videos and storiesprovideyou withanopportunity totellyourstoryin dynamic way. Additionally, thanks to the video options Instagram and modern phones provide, videos have never been easier to make. Which leads to the next point ...

\section{Always edit your photos and video}

There is a lot of competing content on Instagram, so posting amateur-looking photos and video will have your audience swiping to the next post. Spend the time to polish your content and ensure it's eyecatching. Sometimes something as little as adding a filter can yield huge results.

\section{Include a call to action}

Photo captions need to be snappy and catchy, but never forget the power of a call to action. You can do this by asking your audience questions or by creating contests and polls. The more you can keep someone engaged, the more likely they'll go down the sales funnel.

\section{Add a location}

You can tag every post you make with a location, helping you target people in your area. You can be as specific as your practice location, or as general as your city or town.

\section{Don't forget to tag}

If you have a staff member or a product featured in your post, don't forget to tag them in the photo and the caption. This helps your organic reach while doing very little work.

\section{Don't stuff your hashtags}

In most cases, you only ever need to use one or two relevant hashtags in your posts; more could seem forced. Instead, focus on the quality of your caption before you add a hashtag. For example, if your practice is in Calgary you could use \#YYC \#YYCOptometry.

\section{Be authentic}

Don't be afraid to show who you are in your posts. People have a good sense when an Instagram post is simply trying to sell something, so offering a little of yourself can help boost engagement and familiarity with your brand.

\section{Have fun}

Finally, have fun! Instagram is a place where amazing content encourages interaction, so the more fun you have, the more exciting and engaging your content will be. Think fun sunglass photos of the staff or a cool quote about vision.

If you want to see examples of better posts for your practice Instagram feed, follow some of your peers who are doing an amazing job. Here are a few of our favorites: @seeandbeseeneyecare @drmacalpineaurora2020@terrihollandoptometry 